\title{
Study of pattern of feet skin temperature distribution during continuous post-operative epidural analgesia
}

Dmitry Kruglov, Randall Stricker and Kevin Howell

\begin{abstract}
An epidural infusion of local anaesthetic via a catheter provides analgesia during and after major surgery. If pain relief is not adequate an underperforming epidural will require troubleshooting, resiting or removal. Physical examination (sensory level and evidence of motor block), vital signs (blood pressure and heart rate), pain scores and epidural performance during the surgery might help to identify the reason for catheter failure. An epidurogram (imaging with contrast study) will confirm epidural catheter position, but this investigation is not routinely used.

Injection of local anaesthetic into the epidural space produces sympathetic blockade with a vasodilation effect and predictive increase of skin temperature in distal lower limbs. Infrared thermography of patients with effective epidural blockade shows increased temperature of the feet. We studied five post-operative epidural infusions on the day of placement and the following two days.

Analysis of thermal imaging obtained with a calibrated PTi120 Fluke camera suggested that the skin temperature distribution is continuous. We have noticed patterns with a positive temperature gradient toward toes (presence of sympathetic block), and with significant negative temperature gradient toward toes (absence of sympathetic block). Also, the shape of the area covered by the highest isotherms could be of diagnostic value.
\end{abstract}

\section{An updated, more complete version.}

Aim

We studied (feet) skin temperature in patients who received thoracic epidural analgesia for pain relief during and after laparotomy (major abdominal surgery). The aim of this pilot was to identify a thermal signature of an effective pain-relieving epidural block.

\section{Materials and methods}

Five consecutive thoracic epidural infusions were followed post-operatively on the day of surgery and for the next two days. All patients were female aged 52-80 years. Levels of epidural placement were: T8 to L2. All patients received standard post-op monitoring. Pain scores (scale from 0 to 4 ) and motor blockade (scale from 0 to 3 ) were recorded. All patients gave consent for thermal imaging.

Images were acquired by calibrated Fluke PTi120 camera at the bedside. Assessment of radiometric data was performed with the use of Fluke software. We took into account skin temperature values 
(in respect to normal body temperature), symmetry of temperature fields (between right and left sides) and the post-surgical day.

\section{Results}

Analysis revealed dynamically changing skin temperature in all patients with identifiable patterns corresponding to clinical performance of epidural analgesia.

Our cohort naturally splits into three subgroups:

1. Good working epidural providing effective analgesia (good pain relief with 0 or low pain scores, allowing early mobilisation, breathing and coughing without pain)

2. Partially working epidural due to laterality of the block (unequal block with better relief on one side)

3. Failed or equivocal epidural: unclear benefit, absence of reliable signs of epidural sympathetic block during surgery (low stable heart rate and blood pressure), and the need for extra intravenous opioid analgesia during and after operation.

The first group includes Cases 1 and 3. These patients demonstrated equally distributed temperature in the whole dorsal foot skin surface on the first and the second day, often with small positive temperature gradient towards the tips of toes: Case 1 (maximum temperatures values of 33.5/35.7C - right and 32.1/35.2C - left, $1^{\text {st }} / 2^{\text {nd }}$ day respectively) and Case $3(37.1 / 34.8 \mathrm{C}$ and $36.8 / 35.5 \mathrm{C}$ ). Clinically these patients had signs of epidural block during surgery and good pain relief with stable low infusion rate (of 5-6 $\mathrm{ml} / \mathrm{hr}$ ) of $0.1 \%$ bupivacaine with $2 \mathrm{mcg} / \mathrm{ml}$ fentanyl.

The second group includes Case 2. It showed the above described pattern on the right foot only for all three days: $36.7 / 36.3 / 35.4 \mathrm{C}$. On the left side there was a "protuberance appearance" of a longitudinal (pointing to the middle toes) narrow triangle shaped isotherm with maximum temperature over the midline of the foot $(34.3 / 32.0 / 32.1 \mathrm{C})$ and less temperature values on the sides. This clinically corresponded to an incomplete analgesia on the "protuberance" side (left) with signs of more prominent epidural block (anatomically higher sensory level and presence of motor blockade) on the opposite (right) side, where the whole skin was equally warm.

The third group includes Cases 4 and 5. These patients displayed a pattern of "perpendicular (to the limb axis) demarcation" (a clear line of a big negative gradient), which divides warm proximal skin area and cold toes. In these group anaesthetic records did not show signs of epidural block; patients had high pain scores after the surgery with quick escalation of infusion rate and extra intravenous opioid analgesia. Temperature values were not particularly informative as, more likely, reflected natural recovery from sympathetic post-operative storm, rather than sympathetic block by epidural infusion.

\section{Discussion}

The quality of thoracic peri-operative epidural analgesia improves the quality of pain relief and outcome of major abdominal surgery ${ }^{1}$. Epidural catheters in Anaesthesia are inserted blindly; there is no reliable bedside test to confirm catheter position (within or outside epidural space) and predict efficiency. Epidural sympathetic blockade at thoracic and lumbar levels even with low concentration 
of bupivacaine causes change of skin temperature in thorax and feet ${ }^{2,3}$. Thermal imaging gives valuable diagnostic information about the presence/absence of epidural block and a degree of laterality. This will be used in troubleshooting when epidural analgesia is not satisfactory. An important outcome is an avoidance of arbitrary decision making regarding stopping an epidural infusion without a trial of catheter manipulation, change of infusion rate and recipe, and, if needed a replacement. Incorrectly chosen troubleshooting steps, based on insufficient information, lead to poor results and harm.

Available literature discusses absolute temperature values and their laterality ${ }^{4}$. Temperature distribution patterns were not studied or identified. We described two major patterns of in relation to sympathetic block at the epidural level: complete block with equal temperature distribution over foot skin surface (usually with small increase of temperature at toe tips), and an incomplete block with "protuberance appearance" (a narrow longitudinal band of a higher temperature isotherms over the midline of the dorsal foot surface).

\section{References}

1. Li Y, Dong H, Tan S, Qian Y, Jin W. Effects of thoracic epidural anesthesia/analgesia on the stress response, pain relief, hospital stay, and treatment costs of patients with esophageal carcinoma undergoing thoracic surgery: A single-center, randomized controlled trial. Medicine (Baltimore). 2019;98(7):e14362. doi:10.1097/MD.0000000000014362

2. Freise $H$, Meissner $A$, Lauer $S$, et al. Thoracic epidural analgesia with low concentration of bupivacaine induces thoracic and lumbar sympathetic block: a randomized, double-blind clinical trial. Anesthesiology. 2008;109(6):1107-1112. doi:10.1097/ALN.0b013e31818db16c

3. Hopf HB, Weissbach B, Peters J. High thoracic segmental epidural anesthesia diminishes sympathetic outflow to the legs, despite restriction of sensory blockade to the upper thorax. Anesthesiology. 1990;73(5):882-889. doi:10.1097/00000542199011000-00015

4. http://anesthesiaexperts.com/uncategorized/thermal-imaging-camera-detects-unilateralepidural-blockade/ 


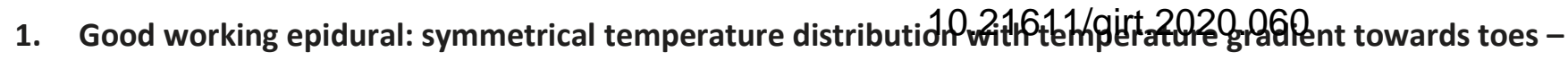
Case $1-1^{\text {st }}$ day

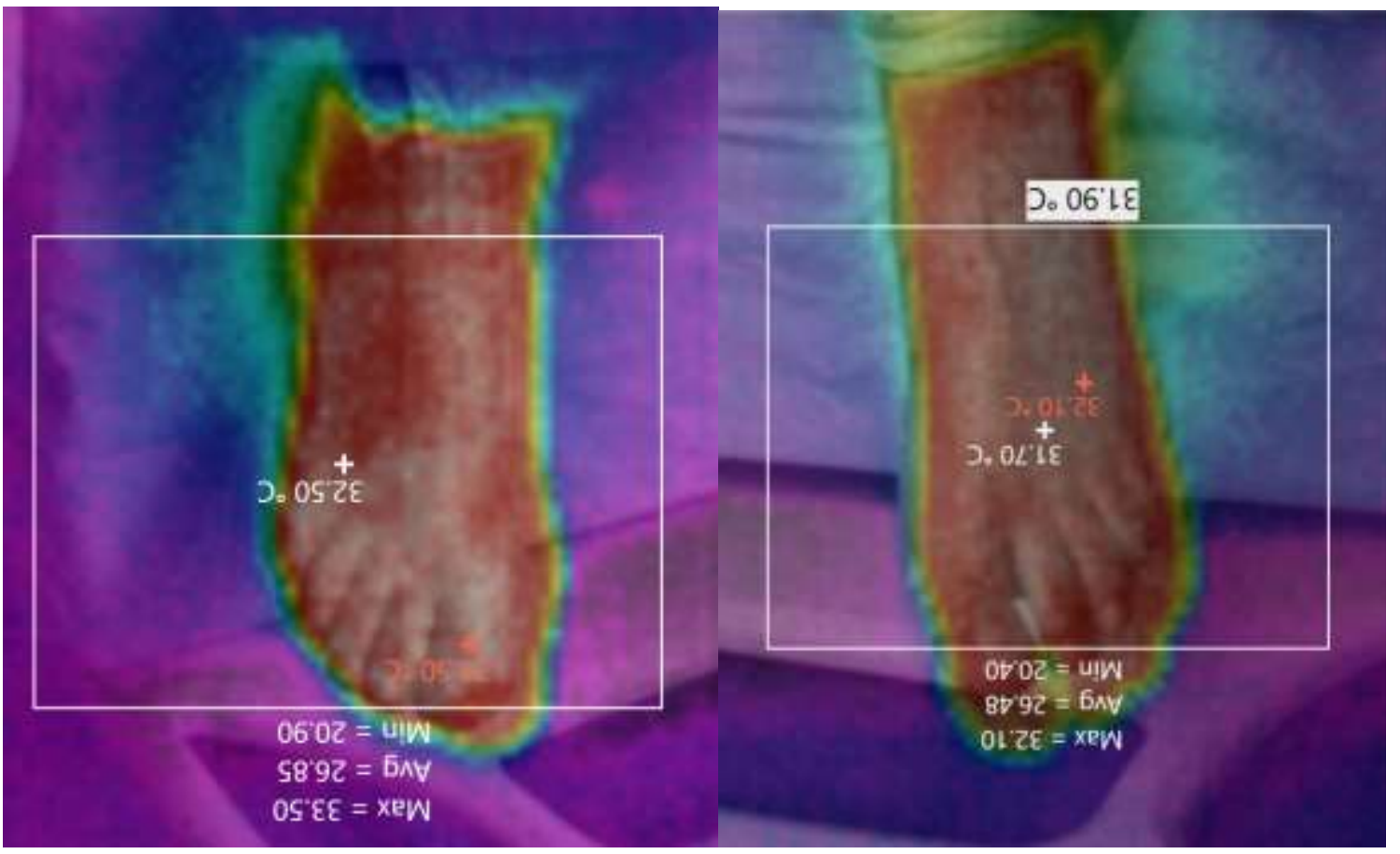

Case 1-day 1- Right and Left

2. Right sided block with "protuberance" pattern on the left - Case $2-1^{\text {st }}$ and $3^{\text {d }}$ days
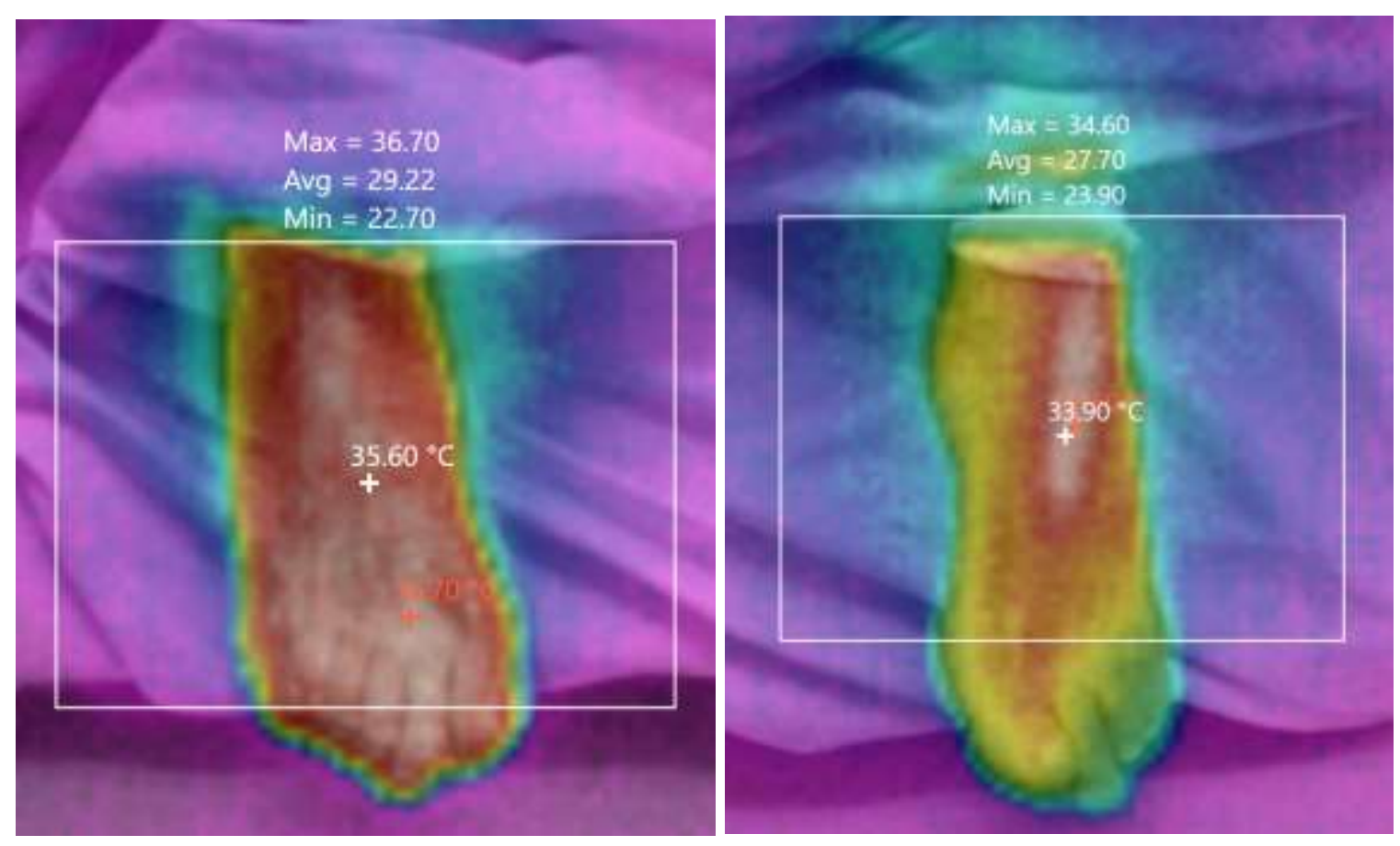

Case 2-day 1- Right and Left 


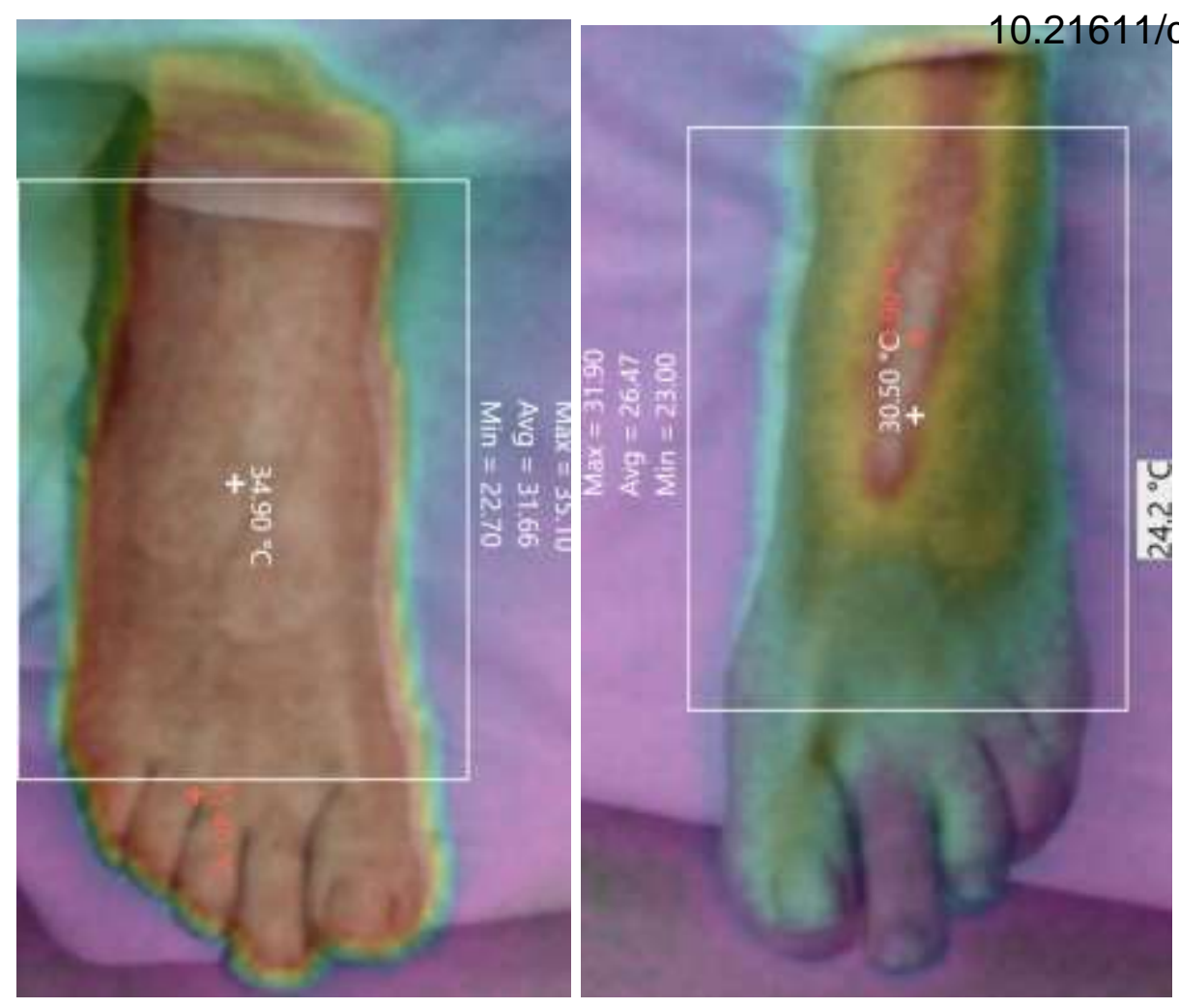

Case 2-day 3- Right and Left

3. No epidural block: "perpendicular demarcation" with cold toes (sharp negative gradient) Case $4-1^{\text {st }}$ day
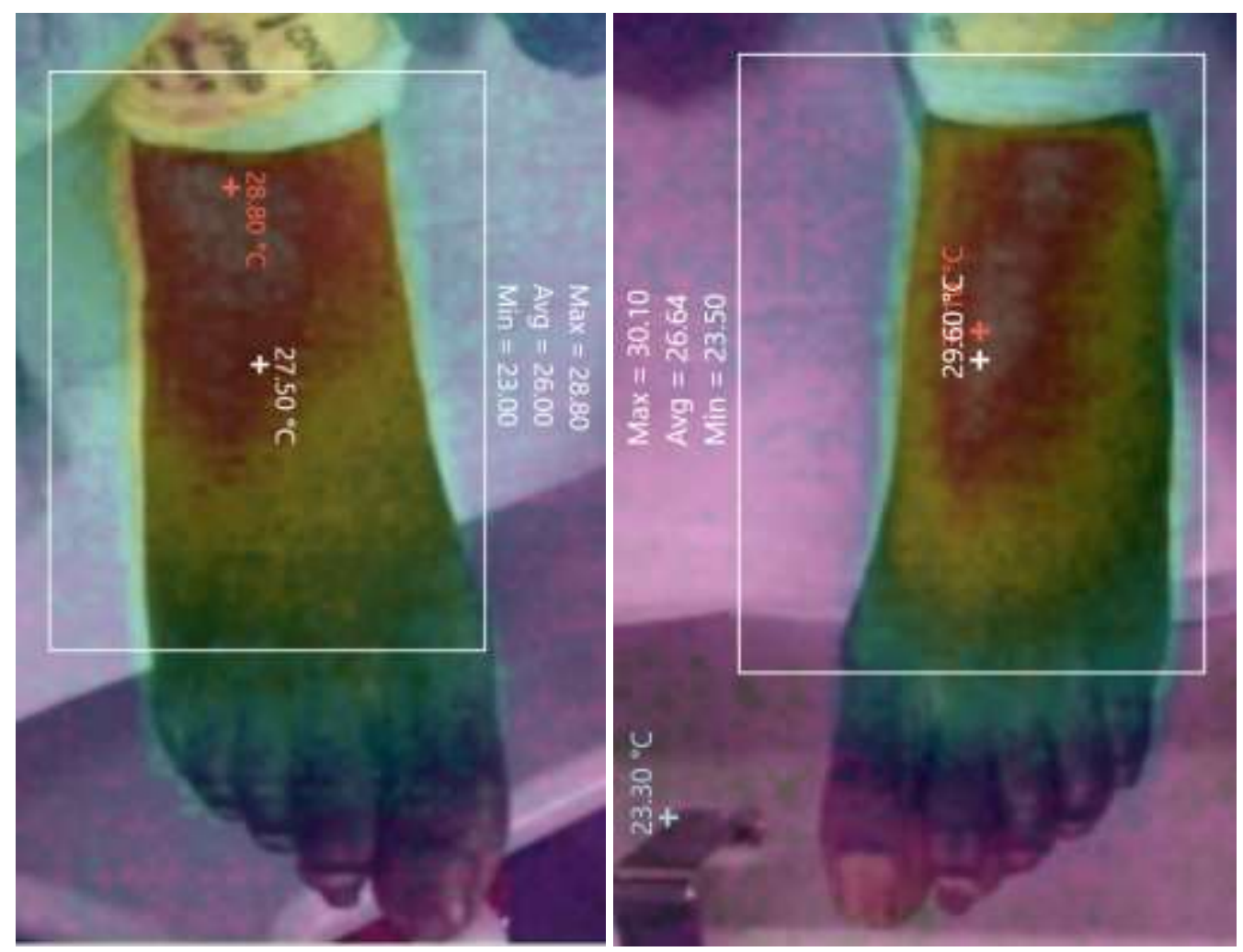

Case4-day1- Right and Left 